\title{
Rastreabilidade das Medições de pH
}

\author{
M. FILOMENA G.F.C. CAMÕES(a) e M. J. GUIOMAR H.M. LITO(b)
}

\author{
A definicaio, recentemente \\ aprovada, de Método Primário de \\ Medicão de $\mathrm{pH}$ aplicada a célula \\ de Hamed, permiliu eslabelecer \\ um método para atribuicaio de \\ valores de pH a Padrōes \\ Primarios de $\mathrm{pH}, \mathrm{PS}$, \\ relacionaveis com a definicion \\ conceptual, $p_{\text {ah }}=\lg _{\text {al }}$. Com \\ base nos trabalhos desenvolvidos \\ pelo grupe de trabalho \\ especialmente designado pela \\ IUPAC, sān tambem apresentadas \\ as Recomendacoues relativas aos \\ Métodos de Certificacão das \\ Solucoóes Padrão Secundárias (SS) \\ comparativamente com as \\ Soluçôes Padrão Primárias (PS), \\ assim como a atribuicão de \\ valores de $\mathrm{pH}$ a solucóes \\ desconhecidas (X), de acordo \\ com os critérios metrológicos. \\ com validade termodinàmica \\ te de forma a construir \\ a rastreabilidade exigivel,
}

\section{INTRODUÇÃO}

A definição da grandeza pH[1], bem como os princípios básicos para a sua medição e determinação. foram estabelecidos e recomendados pela IUPAC em 1985.[2] Alegadas indefinições e incoerências foram surgindo num crescendo que encaminhava para a revisão do documento.

Os desenvolvimentos entretanto obtidos, bem como as discussões científicas a eles associados, levaram a concluir que se atingiu um mais elevado estado de esclarecimento tanto nos aspectos experimentais como teóricos $[3,4,5,6]$.

Desenvolvimentos paralelos no domínio da rastreabilidade das medições químicas impõem a atribuição de incertezas aos valores de grandezas determinadas experimentalmente $[7,8]$.

O presente trabalho dá conta de um estudo profundo e detalhado, apoiado no conhecimento científico, sobre a grandeza $\mathrm{pH}$, consistente com os critérios metrológicos aceites internacionalmente, levado a cabo por um grupo "ad-hoc" nomeado pela IUPAC e desenvolvido com base nos trabalhos de investigadores, técnicos e utilizadores de quase um século.

Este documento é uma adaptação de outro, esse sim a recomendação IUPAC [9], em finalização e que será proximamente publicado no órgão oficial, Journal of Pure and Applied Chemistry. Ele revogará recomendações anteriores e constituirá um documento base a partir do qual outros se poderão desenvolver sobre aspectos específicos relativos a áreas científicas complementares, como seja o desenvolvimento de novos modelos de soluções electrolíticas que possam com vantagem substituir a Convenção de Bates-Guggenheim, para alargamento a outros domínios de aplicações diversificadas: aplicações fisiológicas, meios de elevada força iónica, chuvas ácidas.

\section{FUNDAMENTOS}

A acessibilidade do equipamento para medições de $\mathrm{pH}$, o seu significado em termos da acidez de um sistema e a sua importância no controlo de múltiplos equilíbrios químicos, contribuem para que o $\mathrm{pH}$ seja o parâmetro químico mais medido. Torna-se, portanto, de extrema importância clarificar conceitos e procedimentos, bem como definir a incerteza de valores obtidos por via experimental.

Foram os trabalhos de Sørensen, no início do século XX [10], sobre a hidrólise enzimática das proteínas, que o levaram a demonstrar a importância vital do $\mathrm{pH}$ no controlo dos processos enzimáticos. Foi ele que introduziu o conceito de "tampão" e exprimiu a concentração dos hidrogeniões em termos de uma potência de dez e simbolizou o termo "expoente do ião hidrogénio" por $\mathrm{pH}$, $c_{\mathrm{H}^{+}}=10^{-\mathrm{pH}}$. Ambos os conceitos foram imediatamente aceites pela comunidade científica, reconhecida que foi a sua enorme importância.
Posteriormente foi evidenciado que a metodologia potenciométrica recomendada para a sua determinação [11] conduziria antes a $a_{\mathrm{H}^{+}}$, pelo que foi considerado mais conveniente definir $\mathrm{pH}$ em termos da actividade dos iões hidrogénio em solução, $\mathrm{pH}=-\lg a_{\mathrm{H}^{+}}$, ou

$$
\mathrm{pH}=-\lg \frac{a_{\mathrm{H}^{+}}}{m^{0}}=-\lg \frac{m_{\mathrm{H}^{+}} \gamma_{\mathrm{H}^{+}}}{m^{0}}
$$

onde $a_{\mathrm{H}^{+}}$e $\gamma_{\mathrm{H}^{+}}$representam respectivamente a actividade e o coeficiente de actividade molal dos iões hidrogénio à molalidade $m_{\mathrm{H}^{+}} \mathrm{e} m^{\mathrm{O}}$ representa um estado padrão referente a $1 \mathrm{~mol} \mathrm{~kg}^{-1}$ de iões hidrogénio.

Trata-se de uma definição conceptual, dado que a actividade de uma única espécie iónica é imensurável, por imaterializável. A sua avaliação depende, pois, da adopção de convenções. Os valores de pH não podem ser determinados com base em medidas isoladas, mas requerem métodos aproximados, para obter o coeficiente de actividade da espécie iónica singular, $\gamma_{\mathrm{H}^{+}}$.

A partir de 1940 a análise instrumental assumiu cada vez maior importância, devido às exigências crescentes relativamente à sensibilidade, velocidade e economia dos métodos analíticos, tendo nos últimos anos os aparelhos medidores de $\mathrm{pH}$ digitais permitido uma reprodutibilidade de valores ao milésimo. No entanto, o progresso associado à definição da grandeza não tem acompanhado o desenvolvimento dos aparelhos medidores.

O significado dos valores medidos está relacionado não só com a precisão dos instrumentos de medida mas também com as soluções padrão que são usadas para os calibrar. Os instrumentos de medida de elevada precisão estão dependentes de metodologias comparativas que requerem calibração e validação com Materiais Padrão rastreáveis.

Embora cerca de noventa anos tenham passado desde que Sørensen (1909) introduziu o conceito de $\mathrm{pH}$, e tendo já sido atingidas sensibilidades e velocidades de 
resposta, na análise instrumental, não imagináveis há poucos anos atrás, novas interrogações se põem relativamente à "rapidez" de execução e "rastreabilidade" de resultados para os novos níveis [12].

As determinações de $\mathrm{pH}$, segundo os processos convencionais, têm sido preocupação não só dos Institutos Metrológicos que têm vindo a impor várias exigências de rigor, como de outros Organismos Internacionais que têm emanado Recomendações entre as quais se salientam, pela sua ampla aceitação, as da IUPAC [2] actualmente em revisão.

Os valores de pH de soluções desconhecidas, são determinados usando Soluções Padrão como referência.

Aos valores de pH (PS) das Soluções Padrão Primário exige-se que tenham valores fixos, independentes dos de outras soluções.

A atribuição dos valores de $\mathrm{pH}$ às Soluções Padrão Primário (PS) é feita pela aplicação do Método Primário através da cadeia de etapas que ligam medições de diferenças de potencial de células de Harned, com a equação de Nernst e com o modelo de Bates-Guggenheim para avaliação do coeficiente de actividade do ião cloreto.

A rastreabilidade vincula a relação entre o valor de $\mathrm{pH}$ medido, $\mathrm{pH}(\mathrm{X})$, e o valor de $\mathrm{pH}$ (PS) do Padrão Primário através de uma cadeia ininterrupta de comparações, especificando as respectivas incertezas.

\section{CÉLULA DE HARNED PARA ATRIBUIÇÃO DE VALORES DE pH A SOLUÇÕES TAMPÃO PADRÃO}

\subsection{Método Primário}

Um Método Primário de medição é um método com a máxima qualidade metrológica, requerendo que todas as operações possam ser completamente descritas e compreendidas. Qualquer limitação do modelo teórico ou da determinação experimental deve-se reflectir no cálculo da incerteza total do método, em termos de unidades do SI.

Um Método Primário pode ser

- directo: mede o valor desconhecido de uma grandeza sem necessidade de referência a um padrão da mesma quantidade (ex.: gravimetria ou coulometria)

- relativo: mede a relação entre o valor desconhecido de uma grandeza e o de um padrão da mesma grandeza; o sinal analítico medido e a grandeza em causa devem ser completamente descritos por uma equação, "a lei analítica".

Um Método Primário directo pode ser combinado com um Método Primário relativo para conduzir a medidas que assegurem características primárias.

A célula de Harned que permite a medição de diferenças de potencial, é uma célula sem transporte com um eléctrodo de hidrogénio, com o hidrogénio seco e à pressão de 1 atm e outro de referência de prata-cloreto de prata, com concentrações do ião cloreto variáveis.

$\mathrm{Pt} \mid \mathrm{H}_{2}\left(\mathrm{~g}\right.$, latm) | Solução tampão (PS), $\mathrm{Cl}^{-}\left(m_{\mathrm{Cl}^{-}}\right) \mid \mathrm{AgCl}, \mathrm{Ag}$

A aplicação da equação de Nernst a esta célula , com a diferença de potencial $E$, é traduzida pela equação:

$E=E_{\mathrm{Ag}, \mathrm{AgCl}}^{0}-\left(\frac{R T}{F} \ln 10\right) \lg \left(m_{\mathrm{H}^{+}}+m_{\mathrm{Cl}^{-}}-\gamma_{\mathrm{H}^{+}} \gamma_{\mathrm{Cl}^{-}}\right)$

ou

$-\lg \left(a_{\mathrm{H}^{+}} \gamma_{\mathrm{Cl}^{-}}\right)=\frac{E-E_{\mathrm{Ag}, \mathrm{AgCl}}^{0}}{\frac{R T}{F} \ln 10}+\lg m_{\mathrm{Cl}^{-}}$

onde $E^{0} \mathrm{Ag} \cdot \mathrm{AgCl}$ é o potencial padrão do eléctrodo de prata-cloreto de prata, determinado com a célula de Harned contendo soluções diluídas de $\mathrm{HCl}$ e $\gamma_{\mathrm{Cl}^{-}}$é o coeficiente de actividade do ião cloreto.

A célula de Harned, reconhecida como altamente reprodutível, satisfaz os requisitos para ser recomendada no Método Primário directo de determinação de $-\lg \left(a_{\mathrm{H}^{+}} \gamma_{\mathrm{Cl}^{-}}\right)$, em soluções aquosas, grandeza expressa integralmente em termos de quantidades ou conhecidas ou determinadas experimentalmente [13].

Procedendo à representação gráfica de $-\lg \left(a_{\mathrm{H}^{+}} \gamma_{\mathrm{Cl}^{-}}\right)$em função de $m_{\mathrm{Cl}^{-}}$e extrapolando para $m_{\mathrm{Cl}^{-}}=0$, para remover o efeito do cloreto adicionado, o valor limite desta função a uma determinada força iónica $I$, pode ser expresso $[14,15]$ como

$$
-\lg \left(a_{\mathrm{H}^{+}}+\gamma_{\mathrm{Cl}^{-}}\right)=\mathrm{p}\left(\mathrm{a}_{\mathrm{H}^{+}} \gamma_{\mathrm{Cl}^{-}}\right)^{0} .
$$

A introdução de uma convenção para o coeficiente de actividade iónica do ião cloreto, $\gamma_{\mathrm{Cl}^{-}}$, permite chegar ao $\mathrm{pH}$

$$
\mathrm{pH}=\mathrm{p} a_{\mathrm{H}}=\mathrm{p}\left(a_{\mathrm{H}} \gamma_{\mathrm{Cl}}\right)^{0}+\lg \gamma_{\mathrm{Cl}}
$$

onde a escrita das cargas é eliminada por uma questão de simplicidade.

A convenção referida, denominada de Bates-Guggenheim tem por

base a equação de Debye-Hückel

$$
\lg \gamma_{\mathrm{i}}=-\frac{A I^{1 / 2}}{1+B a_{\mathrm{i}} I^{1 / 2}}
$$

onde $I$ é a força iónica do meio expressa em mol kg-1, $I=\frac{1}{2} \sum_{i} \mathrm{~m}_{\mathrm{i}} z_{i}^{2}$, sendo $z_{\mathrm{i}}$ o número de carga de cada ião e $m_{\mathrm{i}}$ a correspondente molalidade.

Convenciona-se para o parâmetro iónico $B a_{\mathrm{i}}$ o valor $1,5 \mathrm{~mol}-1 / 2 \mathrm{kgl}^{1 / 2}$ para todas as temperaturas, com $a_{\mathrm{i}}=4,6 \AA$ a $25^{\circ} \mathrm{C}$

$$
\lg \gamma_{\mathrm{Cl}}=-\frac{A I^{1 / 2}}{1+1,5 I^{1 / 2}}
$$

$A$ e $B$ são constantes da equação de Debye-Hückel que dependem da temperatura e da permitividade relativa, $\varepsilon_{\mathrm{r}}$, do solvente $\left(\varepsilon_{\mathrm{r}}=\varepsilon / \varepsilon_{0}\right.$, sendo $\varepsilon_{0}$ a permitividade do vazio), 
$A$ é expresso em $\mathrm{mol}^{-1 / 2} \mathrm{~kg}^{1 / 2}$ e $B$ em $\mathrm{mol}^{-1 / 2} \mathrm{~kg}^{1 / 2} \mathrm{~cm}^{-1}$.

Para forças iónicas até $0,1 \mathrm{~mol} \mathrm{kg-1}$, a incerteza padrão** associada ao cálculo do $\mathrm{pH}$ (PS) é de $\pm 0,005$ unidades, mesmo se $B a_{\mathrm{i}}$ na equação de Debye-Hückel variar de $10 \%$ [13].

Os valores de $\mathrm{pH}$ assim obtidos são convencionalmente adoptados como valores de referência, $\mathrm{pH}$ (PS).

Pode-se afirmar que a célula de Harned associada à utilização da Convenção de Bates-Guggenheim é a base de um Método Primário de Referência para a atribuição de valores de $\mathrm{pH}$ a Padrões Primários.

Dado o irrealismo da definição de $\mathrm{pH} \quad\left(\mathrm{pH}=-\lg a_{\mathrm{H}^{+}}\right)$, que envolve a actividade do ião hidrogénio como ião singular e não existindo nenhum método experimental que permita o estabelecimento de valores perfeitamente concordantes com os valores teóricos, recomenda-se o uso do termo "convencional " para explicitar que esta definição não pode ser usada para medir o $\mathrm{pH}$.

A aplicação de um método primário à determinação de $\mathrm{pH}$ de uma solução tampão, que obedeça aos critérios de qualidade metrológica para soluções padrão, faz dela um Padrão Primário de $\mathrm{pH}$.

A figura 1, ilustra em forma esquemática os passos envolvidos na atribuição de valores de $\mathrm{pH}$ (PS).

3.2. Soluções Padrão Primário e as suas Incertezas

Para que as medições de $\mathrm{pH}$ com a célula de Harned sejam rastreáveis às unidades do sistema SI, a incerteza de cada passo deve ser incluída no resultado [16].

O Método Primário para a atribuição de valores "convencionais" de pH deve explicitar as incertezas devidas ao uso da convenção de Bates Guggenheim e as da extrapolação para $m_{\mathrm{Cl}}=0$ que conduz ao $\mathrm{pH}$. Qualquer melhoramento no cálculo de $\gamma_{\mathrm{Cl}}$, por exemplo a utilização do modelo de Pitzer [8], deve contribuir para minimizar as incertezas devidas à convenção de Bates - Guggenhein e aumentar a aplicação do Método Primário a outras soluções de força iónica superior.

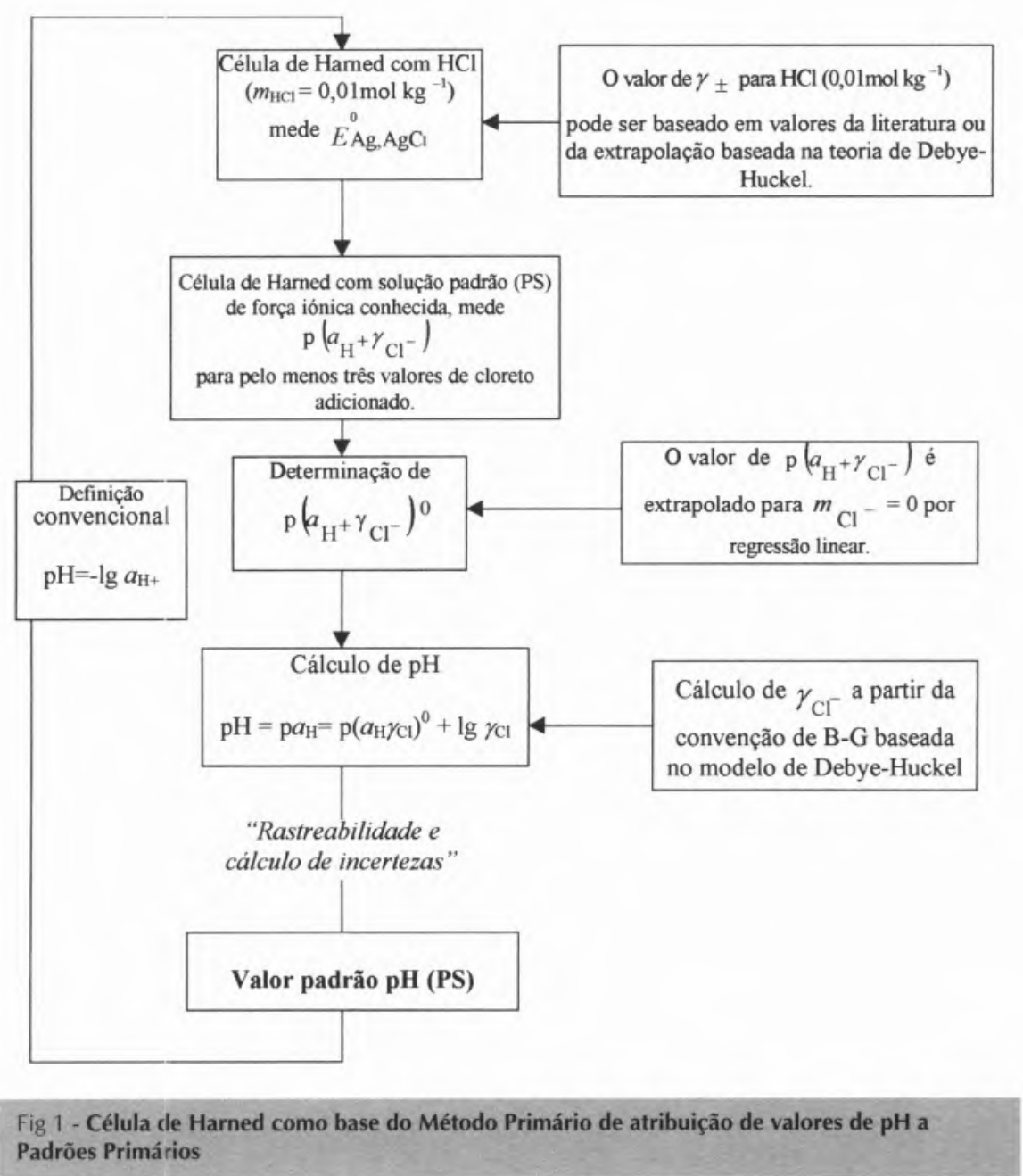

Para que uma solução tampão possa ser considerada Solução Padrão Primário de pH (PS) é necessário não só que o valor de $\mathrm{pH}$ seja determinado pelo Método Primário mas, antes ainda, que satisfaça também o critério de possuir as mais elevadas qualidades metrológicas. O grau metrológico de uma Solução Padrão depende da sua posição na cadeia de rastreabilidade, figura 2 .

Para ser considerada uma Solução Padrão Primário a sua incerteza deve ser considerada a menor possível para que não influencie significativamente a incerteza da Solução Padrão de grau imediatamente inferior, Padrão Secundário. Na prática, os critérios a considerar são:

i) Elevado estado de pureza do Material de Referência Certificado para a preparação da solução,

ii) Elevada reprodutibilidade de valores de $\mathrm{pH}$,

iii) Boa capacidade tampão,

iv) Ligeira dependência do $\mathrm{pH}$ com a temperatura,

v) Ligeira variação do $\mathrm{pH}$ com a concentração,

vi) Aplicabilidade da convenção de Bates - Guggenheim.

vii) Demonstração da rastreabilidade do método.

O Método Primário de medida de $\mathrm{pH}$, usando a célula de Harned foi usado em ensaios interlaboratoriais internacionais, (incluindo, em Portugal, o CECUL e a Universidade da Beira Interior) com a finalidade de avaliar a qualidade das medidas praticadas, em termos de exactidão do Método 


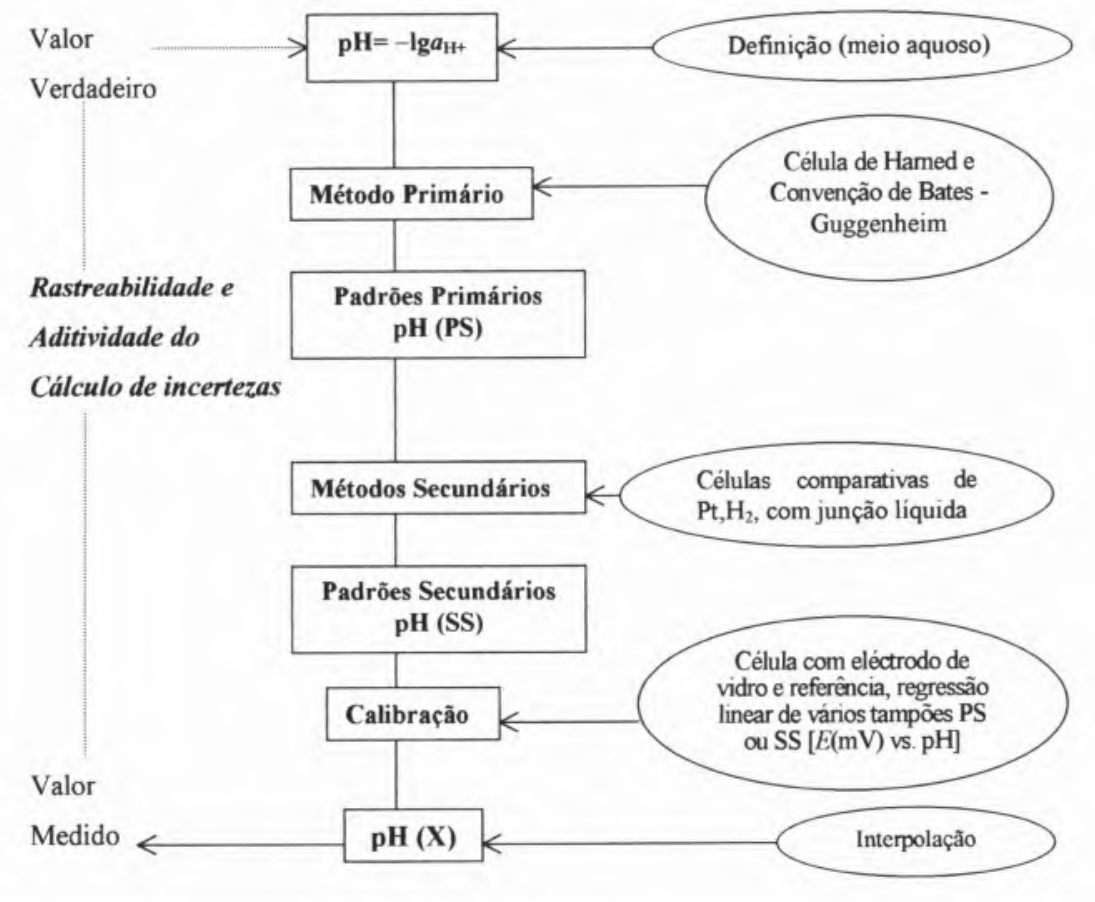

Fig 2 - Cadeia de rastreabilidade nas medidas de $\mathrm{pH}$

Primário para a determinação do $\mathrm{pH}$ das soluções tampão. A intercomparação dos vários ensaios, integrada num programa de controlo de qualidade, patrocinado pelo EUROMET $[17,18]$ demonstrou a comparabilidade das medidas em diferentes laboratórios, com amostras do mesmo lote e permitiu concluir que não existiam diferenças experimentais significativas entre os resultados, a um nível de confiança de $95 \%$. As variações nos valores de $\mathrm{pH}$ das várias amostras não eram superiores a 0,003 . No entanto recomenda-se que o resultado da medida de $\mathrm{pH}$ com a célula de Harned dos Padrões Primários deva incluir a combinação das incertezas de cada passo, a que corresponde uma incerteza expandi$\mathrm{da}^{\star \star *}$ de $\pm 0,01$ associada ao valor de $\mathrm{pH}$, numa gama de 3 a 11 .

As possíveis incertezas do $\mathrm{pH}$ dos Padrões Primários devem ser atribuídas fundamentalmente a dois factores:

- incertezas experimentais, provenientes das variações da pureza dos materiais,

- inconsistências resultantes da aplicação da Convenção de Bates -

Guggenheim a uma variedade de tampões quimicamente diferentes e de diferentes forças iónicas (sempre inferiores a $0,1 \mathrm{~mol} \mathrm{~kg}^{-1}$ ).

\section{MÉTODOS SECUNDÁRIOS E PADRÕES SECUNDÁRIOS}

As soluções de materiais de referência com composição diferente dos Padrões Primários, cujo $\mathrm{pH}$ possa ser medido com o Método Primário, com os resultados rastreáveis aos Métodos Primários, mas com incertezas superiores às determinadas para as Soluções Padrão Primário classificam-se de Soluções Padrão Secundário (SS).

São ainda Padrões Secundários as soluções cujos valores de $\mathrm{pH}$ determinados por Métodos Secundários e rastreáveis relativamente aos Métodos Primários segundo Recomendações que actualmente estão a ser revistas, possam ser comparados com os das soluções Padrão Primário.

Pt | $\mathrm{H}_{2}$ (g, latm) | Solução Tampão SS || Solução Tampão PS | (g, latm) $\mathrm{H}_{2} \mid \mathrm{Pt}$

Contrariamente ao Método Primário em que a célula de Harned é a única proposta para a determinação do $\mathrm{pH}$, várias células que permitam medir a diferença de $\mathrm{pH}$ entre duas soluções tampão têm sido propostas, como base de Métodos Secundários . Os Métodos Secundários estão afectados de incerteza mais elevada que a incerteza associada à atribuição de valores $\mathrm{pH}$ (PS).A escolha da célula depende da incerteza admissível e dos recursos disponíveis.

Para obtenção do valor de $\mathrm{pH}$ de um Padrão Secundário, SS, recomenda-se o uso da célula que contém dois Padrões, PS, certificado e SS, com uma junção líquida, (II), ou com duas junções líquidas numa ponte salina de $\mathrm{KCl}$ (saturado ou de concentração elevada) (III), ou ainda o uso consecutivo de duas células contendo os Padrões PS e SS, respectivamente, com uma junção líquida em cada célula (IV).

A célula (II) pode ser usada para determinar o pH de uma solução Padrão Secundário, SS, com composição química nominal e concentração semelhante ao Padrão Primário, PS, e em que a diferença de $\mathrm{pH}$ não exceda 0,02 [19].

A diferença de $\mathrm{pH}$ medida é: $\mathrm{pH}(\mathrm{SS})-\mathrm{pH}(\mathrm{PS})=E_{\mathrm{II}} / k$

em que $k=R T(\ln 10) / F$, sendo $R$ a constante dos gases, $T$ a temperatura termodinâmica e $F$ a constante de Faraday.

Em situações diferentes da especificada anteriormente recorre-se à célula (III). O Padrão Primário, PS, deverá ser de força iónica e pH próximos do Padrão Secundário, SS. Esta célula poderá também ser usada para medir os valores de Padrões Secundários que não são compatíveis com o eléctrodo de prata-cloreto de prata usado na célula (I). Pode também ser usada para Padrões Secundários com concentrações diferentes e /ou força iónica superior ao limite imposto pela convenção de BatesGuggenheim $\left(I \leq 0,1 \mathrm{~mol} \mathrm{~kg}^{-1}\right)$. 


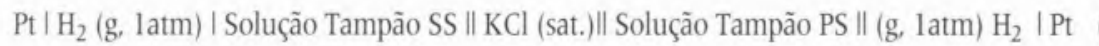

(III)

$\mathrm{pH}(\mathrm{SS})-\mathrm{pH}(\mathrm{PS})=\left[E_{\mathrm{III}}\left(\varepsilon_{\mathrm{j} 2}-\varepsilon_{\mathrm{j} 1}\right)\right] / k$

A diferença de $\mathrm{pH}$ inclui os termos $\varepsilon_{\mathrm{j} 1}$ e $\varepsilon_{\mathrm{j} 2}$ que são os potenciais de junção líquida (representada pelo símbolo II) originados pela junção entre a solução de $\mathrm{KCl}$ (sat.) e as soluções PS e SS respectivamente e que não podem ser quantificados.

Quando os Padrões Secundários têm uma composição química diferente da dos Padrões Primários é usada a célula (IV). Esta célula inclui um eléctrodo de hidrogénio e um eléctrodo de referência com uma ponte salina, geralmente de um electrólito, como o cloreto de potássio, saturado ou de concentração elevada $\left(\geq 3,5 \mathrm{~mol} \mathrm{~kg}^{-1}\right)$.

Ag | AgCl | KCl (sat.) || Solução Tampão SS | (g, latm) $\mathrm{H}_{2}$ | Pt

O valor de $\mathrm{pH}$ é calculado a partir da diferença de potencial medida, $E_{\mathrm{IV}}$, através da equação:

$\mathrm{pH}(\mathrm{SS})=-\left[E_{\mathrm{IV}}(\mathrm{SS})-E_{\mathrm{IV}}^{0}+\varepsilon_{\mathrm{ji}}\right] / k$

em que $E_{\text {IV }}^{0}$ é o potencial padrão do eléctrodo de referência.

Em substituição do eléctrodo de prata-cloreto de prata pode ser usado outro eléctrodo de referência, como o de calomelanos.

Alternativamente pode ainda recorrer-se a duas medições consecutivas, $E_{\mathrm{IV}}\left(\mathrm{SS}_{1}\right)$ e $E_{\mathrm{IV}}\left(\mathrm{SS}_{2}\right)$, na célula (IV).

A correspondente diferença de $\mathrm{pH}$ é dada por:

$\mathrm{pH}\left(\mathrm{SS}_{2}\right)-\mathrm{pH}\left(\mathrm{SS}_{1}\right)=-\left[E_{\mathrm{IV}}\left(\mathrm{SS}_{2}\right)-\right.$

$\left.-E_{\mathrm{IV}}\left(\mathrm{SS}_{1}\right)\right] / k\left[\varepsilon_{\mathrm{j} 2}-\varepsilon_{\mathrm{j} 1}\right] / k$

onde o termo, [ $\left.\varepsilon_{\mathrm{j} 2}-\varepsilon_{\mathrm{j} 1}\right] / k$, designado por potencial residual de junção líquida, é considerado desprezável.

A célula $(\mathrm{V})$ em que o eléctrodo de hidrogénio gasoso é substituído por outro eléctrodo indicador de hidrogénio, como o eléctrodo de vidro, de preferência só deve ser

usada quando não for possível utilizar a célula (II), por exemplo, se o tampão é reduzido pelo hidrogénio gasoso. Esta célula acarreta um aumento de incertezas. Com ela se pode determinar o $\mathrm{pH}$ de soluções tampão, para as quais existam ou não Padrões Primários. Neste último caso, um Padrão de Referência, S, deve ser seleccionado de modo a minimizar o potencial de junção líquida.

$\mathrm{Ag}|\mathrm{AgCl}| \mathrm{KCl}$ (sat.) || Solução Tampão $\mathrm{S}_{\mathrm{i}=1,2} \mid$ Eléctrodo de vidro

A diferença de $\mathrm{pH}$ é dada por:

$\mathrm{pH}\left(\mathrm{S}_{2}\right)-\mathrm{pH}\left(\mathrm{S}_{1}\right)=\left[E_{\mathrm{V}}\left(\mathrm{S}_{2}\right)-E_{\mathrm{V}}\left(\mathrm{S}_{1}\right)\right] /$ $k^{\prime}-\left[\varepsilon_{\mathrm{j} 2}-\varepsilon_{\mathrm{j} 1}\right] / k^{\prime}$

onde $k^{\prime}$ é o declive prático

$$
k=\frac{E_{\mathrm{V}}\left(\mathrm{PS}_{1}\right)-E_{\mathrm{V}}\left(\mathrm{PS}_{2}\right)}{\mathrm{pH}\left(\mathrm{PS}_{1}\right)-\mathrm{pH}\left(\mathrm{PS}_{2}\right)}
$$

O valor de $k^{\prime}$ deve ser registado em função de pH (PS $)_{i}$ e não é necessariamente igual ao declive teórico $k$, devido aos erros inerentes ao eléctrodo de vidro.

Medidas consecutivas com a célula (V) não devem ser usadas para comparar tampões com diferenças de $\mathrm{pH}$ superiores a 0.5 , uma vez que o declive $k^{\prime}$ é determinado por medidas independentes.

As equações para as células II a $\mathrm{V}$ mostram que estas não podem ser consideradas em Métodos Primários, uma vez que contêm potenciais de junção líquida que não podem ser quantificados. Fazem parte de métodos usados para determinar os valores de $\mathrm{pH}$ de Tampões Secundários relativamente aos Primários. As limi-

$\mathrm{Ag}|\mathrm{AgCl}| \mathrm{KCl}$ (conc.) || Solução (S) ou (X) | Eléctrodo de vidro tações associadas com as equações de medida têm uma maior incerteza para os Padrões Secundários do que para os Padrões Primários.

Quando se medem diferenças de $\mathrm{pH}$ deve ser sempre referido se $\mathrm{S}_{1} \| \mathrm{S}_{2}$ são dois Padrões Secundários ou um Primário e um Secundário, pois se forem dois Padrões Secundários o nível de incerteza é maior.

Embora qualquer das células II a $\mathrm{V}$ possa ser usada para certificar Padrões Secundários, as células II e mais simples, para certificar Padrões Secundários relativamente a Primários da mesma composição química. As células IV, V ou a III são as usadas quando os Padrões Secundários não têm a mesma composição química dos Primários.

\section{CALIBRAÇÃO E MEDIDA DE pH DE SOLUÇÕES DESCONHECIDAS (X)}

A utilização de aparelhos medidores de $\mathrm{pH}$, equipados geralmente com eléctrodos de vidro combinados, ou simples com eléctrodos de referência externa, implica metodologias comparativas que requerem calibração e validação com Materiais de Referência Padrão rastreáveis.

Os métodos de calibração para aplicações laboratoriais dependem do fim para que são usados, da incerteza admissível e dos recursos disponíveis.

As medidas de $\mathrm{pH}$ de soluções desconhecidas (X) são efectuadas em células com um eléctrodo de vidro, ou outros sensíveis à espécie hidrogenião, e um eléctrodo de referência (ex: prata, cloreto de prata) com potencial constante (VI) 
O potencial, E, da célula (VI) é devido aos potenciais dos eléctrodos de vidro e de referência, $\varepsilon_{\mathrm{v}}$ e $\varepsilon_{\text {ref. }}$. respectivamente, e ao potencial de junção líquida, $\varepsilon_{\mathrm{j}}$

$E=\varepsilon_{\mathrm{v}}-\varepsilon_{\text {ref. }}+\varepsilon_{\mathrm{j}}$

Estes potenciais estão sujeitos a vários efeitos sistemáticos e ocasionais que afectam as medidas de $\mathrm{pH}$ :

i. Os eléctrodos de vidro têm uma resposta sub-Nernstiana [20], que pode estar sujeita a efeitos de memória variando com o tempo e o historial do uso.

ii. Os potenciais $\varepsilon_{\mathrm{v}}, \varepsilon_{\text {ref. }}$ e $\varepsilon_{\mathrm{j}}$ são dependentes da temperatura.

iii. Os potenciais de junção líquida variam com a composição das soluções de contacto; têm um valor diferente quando, na célula (VI), uma solução é substituída por outra, (X) ou (S). São também afectados pela geometria da junção líquida. Se a junção, do tipo mistura homogénea, for substituída por uma junção do tipo comercial, como as de cerâmica, de diafragma, a de fibra ou de platina, diminui a reprodutibilidade e aumenta a incerteza expandida.

Devido ao desconhecimento de cada uma destas incertezas, a rastreabilidade da medida de $\mathrm{pH}$ de uma amostra desconhecida requer um processo de calibração adequado.

As medidas de $\mathrm{pH}$ de soluções desconhecidas $(\mathrm{X}), \mathrm{pH}(\mathrm{X})$, podem ser efectuadas por três métodos diferentes:

- usando a célula (VI) contendo a solução $(X)$ e comparando o potencial medido, $E(X)$, com o valor medido, $E(\mathrm{~S})$, na mesma célula com um $\mathrm{Pa}$ drão Primário ou Secundário, (S), de $\mathrm{pH}$ conhecido, $\mathrm{pH}(\mathrm{S})$

$$
\mathrm{pH}(\mathrm{X})=\mathrm{pH}(\mathrm{S})-\frac{E(\mathrm{X})-E(\mathrm{~S})}{k^{\prime}}
$$

- usando a célula (VI) contendo a solução $(\mathrm{X})$ e comparando o potencial medido, $E(\mathrm{X})$, com os valores próximos, $E\left(\mathrm{~S}_{1}\right)$ e $E\left(\mathrm{~S}_{2}\right)$, de dois $\mathrm{Pa}$ drões Primários ou Secundários, $\left(\mathrm{S}_{1}\right)$ e $\left(S_{2}\right)$
$\mathrm{pH}(\mathrm{X})=\mathrm{pH}\left(\mathrm{S}_{1}\right)-\left[\mathrm{pH}\left(\mathrm{S}_{1}\right)-\mathrm{pH}\left(\mathrm{S}_{2}\right)\right] \frac{E(\mathrm{X})-E\left(\mathrm{~S}_{1}\right)}{E\left(\mathrm{~S}_{1}\right)-E\left(\mathrm{~S}_{2}\right)}$

- após calibração por determinação da intersecção e declive do potencial, $E$, da célula (VI) em função do $\mathrm{pH}$, com vários Padrões Primários ou Secundários seguida da análise de regressão linear apropriada. Aqui, outros métodos estatísticos, mais informativos que o método dos desvios mínimos quadrados, especificamente desenvolvido para poucos pontos, podem ajudar a identificar possíveis "outliers" dentro de um conjunto de Soluções Padrão.

Alternativamente, os valores de $\mathrm{pH}$ de soluções tampão podem ser calculados a partir das constantes de dissociação termodinâmica dos ácidos fracos envolvidos, admitindo que estes são conhecidos ou determinados independentemente.

Neste processo de cálculo, as incertezas associadas ao conhecimento dos coeficientes de actividade tendem a ser minimizadas. Com o auxílio de técnicas computacionais foi feita a determinação completa e rigorosa dos parâmetros $(\mathrm{pH}, \alpha, \beta, \gamma, I)$ que condicionam a selecção da Solução Padrão de Referência $[3,5,6,13]$.

A adopção de um modelo válido para avaliação dos coeficientes de actividade das espécies iónicas fornece a base para a cadeia de etapas que ligam medições de diferenças de potencial de células de Harned, com a equação de Nernst e com o modelo de Debye-Hückel ou o de Pitzer, dependendo da força iónica da solução, de forma a constituir a rastreabilidade desejada.

Uma vez compreendido tudo é simples. Nesta perspectiva, foi nossa intenção, nos pontos anteriores, apresentar o tema numa forma sistemática e clara. O leitor familiarizado com este tópico poderá estranhar a ausência de referência a termos, conceitos e metodologias que faziam parte de recomendações anteriores [2]. Assim, "escalas", "células e definições operacionais" são exemplos de termos e conceitos a riscar do glossário de termos relativos a $\mathrm{pH}$, por reconhecidamente inapropriados e indevidamente utilizados no passado.

Essas omissões são uma opção metodológica, precisamente para que se construa a cadeia de rastreabilidade.

Centro de Electroquímica e Cinética

da Universidade de Lisboa

(a) Faculdade de Ciências da Universidade de Lisboa

(b) Faculdade de Farmácia da Universidade

de Lisboa

\section{NOTAS}

* Rastreabilidade é a propriedade do resultado de uma medição ou de um valor de referência que permite o seu relacionamento com padrões geralmente nacionais ou internacionais, através de uma cadeia ininterrupta de comparações, especificando as correspondentes incertezas, até grandeza expressa em unidades do sistema internacional (S.I.) [ 7]. Actualmente, na definição de rastreabilidade, existe uma nova frase que salienta o facto de que a atribuição de incertezas ao resultado de uma medida simples, só é valida num intervalo de tempo limitado; exige-se que haja comparações periódicas directas [ 8] .

** Incerteza padrão é a incerteza de um resultado de medidas expressa como desvio padrão.

*** Incerteza expandida é uma quantidade que define um intervalo no qual se situa, com um certo nível de confiança, o valor da grandeza medida. A incerteza expandida é o produto da incerteza padrāo por um factor de cobertura, função do nível de confiança desejado ( 2 , para um nível de confiança de $95 \%$ ).

\section{REFERENCIAS}

1. Le Comité Consultif pour La Qualité de la Matiére, (1998). 
2. A.K. Covington, R.G. Bates and R. Durst, Pure and Appl. Chem., 57, 531 (1985).

3. M.J.G.H. Lito, M.F.G.F.C. Camões, M.I.A. Ferra and A.K. Covington, Anal. Chim. Acta, 239, 129 (1990).

4. A.K. Covington, M.I. Ferra, J. Sol. Chem., 23, 1 (1994).

5. M. Filomena Camões, M.J. Guiomar Lito, M. Isabel A. Ferra and Arthur K. Covington, Pure and Appl. Chem. 69, 1325 (1997).

6. M.J. Guiomar Lito, M. Filomena G.F.C. Camões, A.K. Covington, J. Sol. Chem., 27(10), 925 (1998).

7. International Vocabulary of Basic and General Term in Metrology, second edition, 1993, BIPM, IEC, IFCC, ISO, IUPAC, IUPAP and IOML, International Organization for Standardization, Geneva (1993).

8. C.D. Ehrlich, S.D. Rasberry, J. Res. NIST, 103, 93 (1998).

9. Relatório em preparação.

10. S.P.L. Sørensen, Biochem Z. 21, 131 (1909).

11. S.P.L. Sørensen and K. Linderstrøm-Lang, Compt. Rend. Trav. Lab. Carlsberg, 15 (1924).

12. C.M. Beck, Analytical Chemistry, 66(4), 224 (1994).

13. M.J. Guiomar Lito, paH de Soluções Tampão de Referência - Desenvolvimento de novas Metodologias Experimentais e Computacionais envolvendo Equilíbrios Químicos, Tese de Doutoramento, Universidade de Lisboa (1991).

14. R.G. Bates, Determination of $\mathrm{pH}$ - Theory and Practice, 2nd edn., Wiley, New York (1973).

15. H.S. Harned, B.B. Owen, The Physical Chemistry of Electrolytic Solutions, 3rd edn.

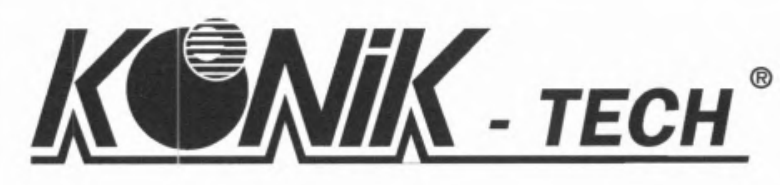

\title{
Kromatografia + EspeKtroscopia
}

\author{
CROMATOGRAFIA: HRGC / HPLC \\ ESPECTROSCOPIA/ESPECTROMETRIA \\ ENGENHARIA \\ EQUIPAMENTOS DE LABORATÓRIO \\ PREPARAÇÃO DE AMOSTRAS \\ CIÊNCIA DE MATERIAIS/VÁCUO
}

\author{
Konik-Tech, S.A. \\ Rua Prof. Veiga Ferreira, 6B \\ 1600 Lisboa \\ Telef. 217573547 \\ Fax. 217573485 \\ E-mail: lisboa@konik-group.com \\ Vendas: sales@konik-group.com \\ Marketing: marketing@konik-group.com \\ Serviço Técnico: SAT@konik-group.com \\ www.konik-group.com
}

Reinhold Publishing Corp., New York (1958).

16. Guide to the Expression of Uncertainty in Measurement (1993), Published in the Name of BIPM,IEC, IFCC, ISSO, IUPAC, OIML by ISO, Geneva.

17. A.K. Covington, M. Filomena Camões and $\mathrm{M}$. Isabel Ferra, "Towards a Unified $\mathrm{pH}$ Scale for the 21 st Century", PTB-Bericht, Vorträge des 126. РTB Seminars: Traceability of
$\mathrm{pH}$ Measurement, Braunschweig, p. 21, 1997.

18. Metrologia 33, 95-96 (1996); 34, 375376 (1997).

19. F.G.K. Baucke, J. Electroanal.Chem. 368 , 67 (1994).

20. F.G.K. Baucke,Anal. Chem. 66, 4519 (1994). 


\section{EQUIPAMENTO DE VÁCUO}

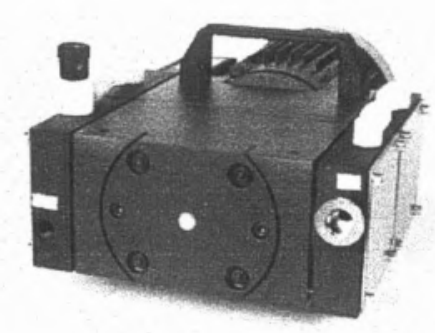

Sistemas de deposição térmica e de Sputtering Detectores de fugas Manipuladores de amostras

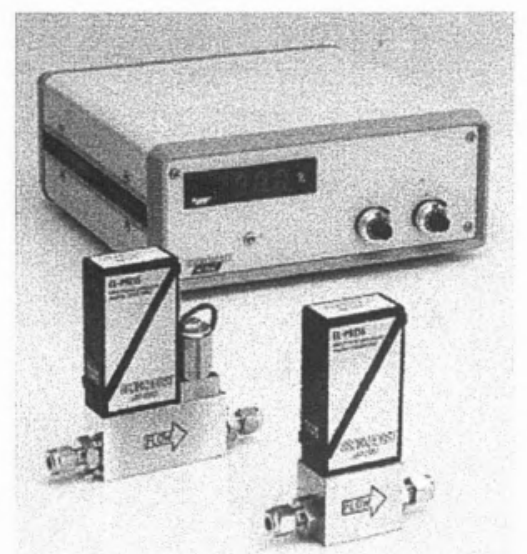

\author{
Bombas de membrana \\ Bombas rotativas a óleo \\ Bombas difusoras \\ Bombas Turbomoleculares \\ Bombas Roots \\ Bombas quimicas secas \\ Bombas lónicas
}

Válvulas, vedantes, flanges,óleos e "greases".

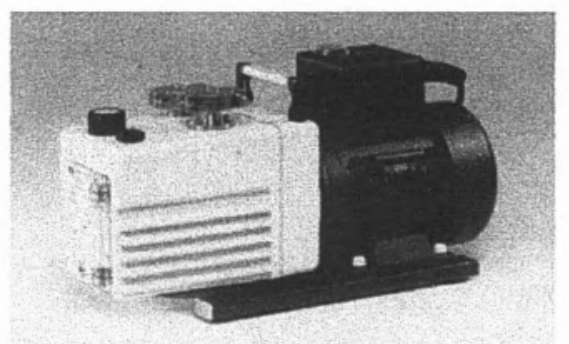

Medidores/controladores de vácuo: - Piezoeléctricos, Piranis, Pennings, Capacitivos (Baratron) e Ion gauges. Medidores/controladores de Fluxo e Massa.

\section{LIOFILIZADORES}

\section{OUTROS EQUIPAMENTOS}

Balanças Analíticas. Banhos de Ultrasons. Banhos Termostáticos. Recirculadores. Geradores de Gases. Manoredutores. Fluxímetros. Manoredutores e Reguladores de Pressão. Compressores Silenciosos. Compressores Secos. Válvulas, Tubos e Coneções para Fluidos.

STV - Equipamentos para a Indústria e Laboratórios, Lda

Casal da Serra, lote 103 - loja Esq.- 2625-082 Póvoa Santa Iria Tel. 01- 9563007 